\title{
Efeitos da manipulação visceral no tratamento da dor em mulheres com dismenorreia primária
}

\author{
Effects of visceral manipulation in the treatment of pain in women with primary dysmenorrhea \\ Efectos de la manipulación visceral sobre el manejo del dolor em mujeres com dismenorrea
}

primaria

Recebido: 08/09/2021 | Revisado: 16/09/2021 | Aceito: 17/09/2021 | Publicado: 18/09/2021

\author{
Jamile Maria de Souza Alves \\ ORCID: https://orcid.org/0000-0002-8132-0940 \\ Universidade Católica de Pernambuco, Brasil \\ E-mail: jamilesouzafisio@gmail.com \\ Roberta Alvarenga Guedes Martins \\ ORCID: https://orcid.org/0000-0003-1159-7300 \\ Universidade Católica de Pernambuco, Brasil \\ E-mail: ralvarengaguedes@gmail.com \\ Thayna Maria Praia Baratella \\ ORCID: https://orcid.org/0000-0003-0890-7259 \\ Universidade Católica de Pernambuco, Brasil \\ E-mail: thaynapraiafta@gmail.com \\ Natália Santos Pereira da Silva \\ ORCID: https://orcid.org/0000-0001-8254-8149 \\ Universidade Católica de Pernambuco, Brasil \\ E-mail: nsantosps2@gmail.com \\ Ana Carolina da Silva Quiros \\ ORCID: https://orcid.org/0000-0001-6407-3282 \\ Universidade Católica de Pernambuco, Brasil \\ E-mail: anacarolinaquiros@hotmail.com \\ Juliana Alves do Monte \\ ORCID: https://orcid.org/0000-0003-2968-5763 \\ Universidade Católica de Pernambuco, Brasil \\ E-mail: julianamontefisio@gmail.com \\ Silvana Maria Macedo Uchôa \\ ORCID: https://orcid.org/0000-0002-4635-9428 \\ Universidade Católica de Pernambuco, Brasil \\ E-mail: silvanammu@gmail.com \\ Paulo Henrique Altran Veiga \\ ORCID: https://orcid.org/0000-0003-4303-8044 \\ Universidade Católica de Pernambuco, Brasil \\ E-mail: paulohveiga1@gmail.com
}

\begin{abstract}
Resumo
Objetivo: O trabalho objetivou avaliar os efeitos da manipulação visceral no tratamento da dor em mulheres com dismenorreia primária. Método: Trata-se de um estudo do tipo ensaio clínico randomizado de corte transversal, no qual foram avaliadas 13 mulheres que apresentavam dismenorreia primária. Inicialmente foi realizada uma coleta de dados sócio clínicos, em seguida foi aplicado um questionário sobre dismenorreia e foi avaliada a intensidade da dor pela Escala Visual Analógica (EVA). Foram divididas em dois grupos sendo um deles o grupo controle (GC) que utilizou a Tens Burst e o grupo experimental (GE) no qual foram realizadas as manipulações viscerais do útero e ovário. Após o fim dos atendimentos as pacientes foram reavaliadas com o mesmo questionário e escala já utilizados. Resultados: Foi possível observar diante dos dados que apesar de ambos os grupos apresentarem resultados positivos depois do período de tratamento, o GE apresentou uma melhora mais significativa quanto a intensidade da dor e redução dos sintomas, quando comparado ao GC após ambas as técnicas, principalmente na fase inicial do ciclo menstrual. Observou-se também no GE uma redução da dor de 3,85 pontos na EVA após a manipulação visceral, já no GC obteve uma redução da dor na EVA de 0,83 pontos ao ser utilizado a TENS. Conclusão: Diante disso, ambas as técnicas apresentaram resultados satisfatórios, no entanto a manipulação visceral apontou resultados mais positivos, principalmente, na fase inicial da menstruação, sendo capaz de diminuir a dor em mulheres com dismenorreia. Palavras-chave: Dismenorreia; Manipulações musculoesqueléticas; Manipulação osteopática; Vísceras.
\end{abstract}




\begin{abstract}
Objective: This study aimed to evaluate the effects of visceral manipulation in the treatment of pain in women with primary dysmenorrhea. Method: This is a cross-sectional randomized clinical trial study, in which 13 women with primary dysmenorrhea were evaluated. Initially, a socio-clinical data collection was performed, then a questionnaire on dysmenorrhea was applied and pain intensity was assessed using the Visual Analog Scale (VAS). They were divided into two groups, one of them being the control group (CG) that used Tens Burst and the experimental group (EG) in which visceral manipulations of the uterus and ovaries were performed. After the end of the consultations, the patients were re-evaluated with the same questionnaire and scale already used. Results: It was possible to observe from the data that although both groups had positive results after the treatment period, the EG showed a more significant improvement in terms of pain intensity and symptom reduction, when compared to the CG after both techniques, especially in the initial phase of the menstrual cycle. It was also observed in the EG a pain reduction of 3.85 points in the VAS after visceral manipulation, whereas in the CG it obtained a pain reduction in the VAS of 0.83 points when using TENS. Conclusion: Therefore, both techniques showed satisfactory results, however visceral manipulation showed more positive results, especially in the initial phase of menstruation, being able to reduce pain in women with dysmenorrhea.
\end{abstract}

Keywords: Dysmenorrhea; Musculoskeletal manipulations; Osteopathic manipulation; Viscera.

\title{
Resumen
}

Objetivo: Este estudio tuvo como objetivo evaluar los efectos de la manipulación visceral en el tratamiento del dolor en mujeres con dismenorrea primaria. Método: Se trata de un ensayo clínico transversal, aleatorizado, en el que se evaluaron 13 mujeres con dismenorrea primaria. Inicialmente se realizó una recolección de datos socioclínicos, luego se aplicó un cuestionario sobre dismenorrea y se evaluó la intensidad del dolor mediante la Escala Visual Analógica (EVA). Se dividieron en dos grupos, siendo uno de ellos el grupo control (GC) que utilizó Tens Burst y el grupo experimental (EG) en el que se realizaron manipulaciones viscerales de útero y ovarios. Una vez finalizadas las consultas, los pacientes fueron reevaluados con el mismo cuestionario y escala ya utilizados. Resultados: Se pudo observar a partir de los datos que aunque ambos grupos tuvieron resultados positivos después del período de tratamiento, el GE mostró una mejoría más significativa en términos de intensidad del dolor y reducción de síntomas, cuando se comparó con el GC después de ambas técnicas, especialmente en el fase inicial del ciclo menstrual. También se observó en el GE una reducción del dolor de 3,85 puntos en la EVA tras la manipulación visceral, mientras que en el GC obtuvo una reducción del dolor en la EVA de 0,83 puntos al utilizar TENS. Conclusión: Por tanto, ambas técnicas mostraron resultados satisfactorios, sin embargo la manipulación visceral mostró resultados más positivos, especialmente en la fase inicial de la menstruación, pudiendo reducir el dolor en mujeres con dismenorrea. Palabras clave: Dismenorrea; Manipulaciones musculoesqueléticas; Manipulación osteopática; Vísceras.

\section{Introdução}

A dismenorreia se caracteriza como cólicas menstruais dolorosas que ocorrem durante a menstruação. Ela pode se apresentar com náuseas, vômitos, diarreia, fadiga, dor lombar, nervosismo, tonturas e cefaleia. A estimativa da prevalência da dismenorreia é de $45 \%$ a $95 \%$ nas mulheres em idade reprodutiva. (Iacovides,2015) Ela pode ser classificada em dois tipos: primária e secundária. A primária é decorrente do aumento da produção e liberação de prostaglandinas durante a menstruação (Claydon e Kannan,2014), enquanto que a secundária possui uma relação com alguma patologia subjacente. (Acqua e Bendlin, 2015)

Na dismenorreia primária ocorre uma hipercontratilidade dos músculos do útero, que é causada pelo aumento da produção e liberação de prostaglandinas, que são compostos endógenos, que abrangem toda a atividade orgânica e tem efeitos na atividade reprodutiva, sendo capaz de alterar a motilidade e mobilidade dos órgãos reprodutores e de provocar o aumento da pressão intra uterina e vasoconstrição dos seus vasos, piorando o quadro de hipóxia e causando dor. (Kramp,2012)

O útero por sua vez, está ligado diretamente aos ovários pelo ligamento útero ovariano, que faz parte dos ligamentos suspensores do sistema urogenital, que são de extrema importância para a mobilidade e função dos órgãos pélvicos. Sendo assim, ao ser realizado uma liberação das restrições dos ligamentos, ocorre diminuição da pressão dos vasos sanguíneos, otimizando a fase vascular e melhorando a eficácia do sistema linfático. E em decorrência disso, ocorre a restauração do fluxo sanguíneo ideal para os órgãos, que repercute em uma melhora na sua função (Kramp,2012)

A manipulação visceral é um método capaz de reorganizar a mobilidade ou motilidade de um órgão de forma sutil utilizando da especificidade do movimento de cada órgão, fornecendo um estímulo ao corpo. Existem três técnicas gerais de 
manipulação, sendo elas: a técnica direta, que atua na mobilidade, na qual é realizado um movimento em direção a correção da disfunção e ao final do movimento é realizada a manipulação; a técnica indireta, que atua também na mobilidade, porém o movimento é realizado em direção a correção e é feito antes de alcançá-la e por fim a técnica de indução que atua na motilidade do órgão, que é definida de acordo com a direção e eixo que o mesmo se movimenta, e é realizada em direção a disfunção. (Barral,2014)

O útero pode ser manipulado de forma direta, indireta ou por indução, no entanto, o ovário deve ser preferencialmente manipulado através da indução por ser um órgão extremamente frágil. $\mathrm{O}$ efeito dessas técnicas tem o intuito de descongestionar e mobilizar o órgão reprodutor, sendo assim, capaz de diminuir ou tratar de fato essa hipercontratilidade do útero gerada pela dismenorreia primária. (Barral,2014)

Diante do exposto, o presente estudo visa observar se houve diminuição da dor e desconforto causados pela dismenorreia primária com o uso da manipulação visceral se baseando nos seus benefícios no útero e no ovário e dessa forma verificar a sua eficácia no quadro clínico desta patologia.

\section{Metodologia}

Este projeto está vinculado a Universidade Católica de Pernambuco, a Escola de Saúde e Ciências da Vida, ao curso de Fisioterapia. É parte integrante do projeto intitulado "Influência da liberação miofascial superficial e profunda na força muscular", aprovado pelo comitê de ética em pesquisa com seres humanos, sobre CAAE: 03095918.9.0000.5206 e parecer de número: 3.085.282. O mesmo foi realizado nos laboratórios de Fisioterapia e Terapia Ocupacional Corpore Sano da referida instituição, localizada na cidade do Recife/PE.

Trata-se de um ensaio clínico randomizado e de corte transversal, no qual a randomização foi realizada de forma que cada indivíduo que se voluntariou para a avaliação, foi colocado em um dos dois grupos de forma aleatória por meio de sorteio. O tamanho da amostra foi estimado em 18 pacientes do sexo feminino com idades entre 18 e 28 anos sendo uma amostra de conveniência, divididos em dois grupos, sendo 9 indivíduos do grupo controle e 9 do grupo intervenção, no período de novembro de 2020 a abril de 2021.

Como critérios de inclusão, foram considerados: mulheres que apresentavam dismenorreia primária que foi identificada, associando a história clínica e a sintomatologia ou exames de imagem, aos quais são considerados diagnóstico diferencial, pois a partir deles identificamos patologias associadas; mulheres cognitivamente competentes; e que aceitaram participar do estudo e assinaram o Termo de Consentimento Livre e Esclarecido (TCLE). Como critérios de exclusão, foram considerados: mulheres que apresentaram dismenorreia secundária que é identificada através da história clínica e exame físico, ou exames de imagem; gestantes; mulheres na menopausa; ou que fazem uso de dispositivo intrauterino (DIU).

Inicialmente, foi realizada uma palestra explicativa na qual foi exposto todos os pontos da pesquisa, a forma como seria feita e os benefícios e riscos do estudo. Em seguida foi apresentado o TCLE, que foi assinado ao haver concordância do indivíduo em participar do estudo.

Todas as participantes preencheram uma ficha de avaliação sócio clínica, desenvolvida pelos pesquisadores, na qual constava os dados pessoais, história clínica e familiar. Em seguida, realizou-se o questionário de dismenorreia baseada no estudo de Lopes,(2016) e no estudo de Rodrigues, (2011), no qual foi aplicado através de uma ficha contendo questionamentos sobre o tipo de dismenorreia,, ou seja, se era primária ou secundária; quanto a intensidade de dor que se apresentava, como leve, moderada ou grave e em que fases do ciclo ocorriam; questões de volume do fluxo, ciclo menstrual, dor, sintomas associados, medicamentos usados e a eficiência dos mesmos durante a dor. E por fim, foi realizada a avaliação da dor através da Escala Visual Analógica (EVA), que consiste em uma escala um de aferição da intensidade de dor de forma subjetiva e referida pela paciente, onde 0 é equivalente a ausência total de dor e 10 é o nível de dor máxima suportável pelo 
paciente (Ciena,2008). A partir desta escala, foi apresentado em uma ficha todas as fases do ciclo menstrual e era quantificada através dos valores dispostos na escala a dor que a participante apresentava em cada fase do ciclo, definindo o seu nível de dor durante o período menstrual.

Após a avaliação, um grupo foi submetido a manipulação visceral do útero e do ovário, pois segundo Barral (2014) esta técnica tem o intuito de descongestionar os órgãos, levando a uma melhora da mobilidade dos mesmos através da manipulação dos próprios órgãos e das estruturas adjacentes a eles, favorecendo o seu desempenho típico. Enquanto que no outro grupo, foi utilizada a estimulação elétrica nervosa transcutânea (TENS) no modo Burst, pois segundo Starkey (2001), consiste em uma técnica de analgesia que altera a percepção da dor com a corrente elétrica através da pele, e em conformidade a isso, seria possível provocar a despolarização dos nervos sensoriais e motores. Além disso, dependendo dos seus parâmetros, seria capaz de causar a diminuição da percepção da dor provocada pela dismenorreia primária, através da teoria das comportas somadas à liberação de beta peptídeos endógenos.

A TENS utilizada foi o do modelo neurodyn da marca Ibramed fabricada no ano de 2020, com frequência de base de $100 \mathrm{~Hz}$ e largura de pulso de 250 microssegundos, com intensidade sub dolorosa por um tempo de 30 minutos. Um par de eletrodos de borracha foi posicionado na coluna lombar na região de L3 e L4 com a paciente em decúbito ventral e em seguida com a paciente em decúbito dorsal, o outro par foi posicionado na região do baixo ventre, nivelado com as espinhas ilíacas ântero superiores. O tratamento foi realizado uma vez por semana durante um mês (Oliveira et al.,2012).

Para a realização da manipulação visceral do útero e ovário, inicialmente foi avaliado a mobilidade destes órgãos, que foi feita com a paciente em decúbito dorsal com o quadril e os joelhos flexionados. A mão do terapeuta se posicionou em cima da espinha ilíaca anterossuperior, empurrando em sentido posterior em direção à sínfise púbica e dessa forma foi avaliada a elasticidade e se havia presença de zonas de aderências. Além disso, também se usou o teste de motilidade, que é um movimento ativo, lento e de amplitude quase imperceptível, tanto do útero como do ovário, no qual o paciente se encontrava em decúbito dorsal com os joelhos flexionados e a mão do terapeuta era posicionada sobre a sínfise púbica e o intuito era sentir o movimento de inspir que ocorre em direção oposta ao eixo mediano e o expir que ocorre em direção ao eixo mediano do corpo, esses movimentos devem ocorrer em sincronia. Quando não ocorrem, há um desequilíbrio entre eles que precisa ser corrigido (Barral, 2014).

Em seguida foi feita a manobra primeiramente do útero, onde foi realizada uma técnica ativa na qual a paciente encontrava-se na posição de trendelenburg invertida e a mão do avaliador localizava-se acima da sínfise púbica e foi feito um movimento translatório do fundo do útero para promover uma liberação local. O tratamento foi finalizado com uma técnica de indução nos ovários, estando a paciente em decúbito dorsal com os membros inferiores flexionados e a mão do terapeuta apresentava-se entre a linha média e a espinha ilíaca superior direita ou esquerda dependendo de qual ovário seria manipulado (Barral, 2014)

Todas as manipulações foram realizadas logo após o período menstrual, uma vez por semana durante 1 mês. A quantidade de manipulações feitas durante o atendimento variava de acordo com a necessidade de cada paciente, com o objetivo de identificar sinais de liberação da mobilidade e motilidade dos órgãos, tendo o intuito de descongestionar e mobilizá-los e consequentemente gerar uma diminuição da dor causada pela dismenorreia.

Após os procedimentos descritos, as pacientes foram novamente submetidas a avaliação através do questionário de dismenorreia e escala EVA e todos os dados foram catalogados em planilha Excel 2016. Ao término da coleta, esses dados foram submetidos a análise estatística para inferência e decisão, dos resultados antes e depois da terapia, nas quais as variáveis contínuas foram apresentadas como média e desvio padrão.

Para testar a suposição de normalidade das variáveis envolvidas no estudo foi aplicado o teste Kolmogorov-Smirnov. Foi verificada parametricidade em todos os dados do estudo. Após a confirmação da mesma, os dados em relação à dor das 
participantes do estudo, foram comparados, através do teste t pareado entre os grupos, e não pareado quando os grupos foram diferentes. Para a discriminação das diferenças da média da EVA, foi utilizado teste $t$ de student pareado, e o teste de Wilcoxon para as medias não normais. Considerou-se o nível de significância $\mathrm{p} \leq 0,05$ e o software utilizado para a obtenção dos cálculos estatísticos foi o GraphPad Prism 4 ® 2005.

\section{Resultados}

Diante a pandemia do novo Coronavírus, 5 participantes das 18 iniciais, não conseguiram dar continuidade aos atendimentos, sendo excluídas da amostra, a qual foi reduzida para 13 mulheres que foram divididas em dois grupos, sendo 6 no grupo controle e 7 no grupo intervenção.

Neste estudo a média de idade das mulheres incluídas foi de 22,00 $\pm 2,00$ anos no grupo TENS e de 21,43 $\pm 1,988$ anos no grupo da Manipulação Visceral. Enquanto que, a média do nível de escolaridade no grupo TENS e no grupo da Manipulação Visceral foi de 5,333 \pm 0,51 sendo elas em sua maioria estudantes com ensino superior, não havendo diferença estatisticamente significativa entre os grupos ( $>00,05)$ (Tabela 1$)$.

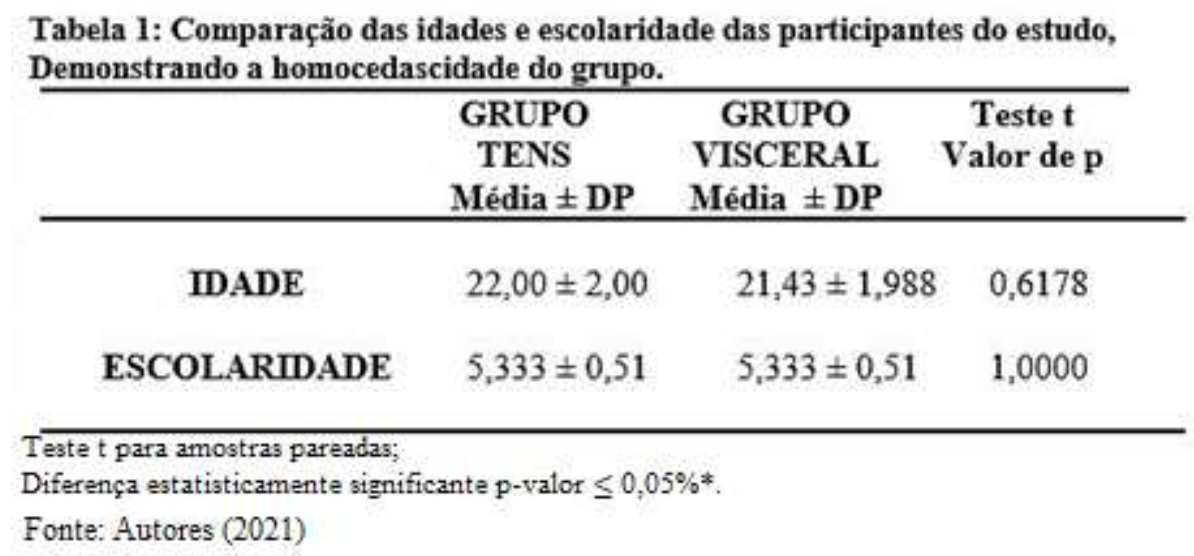

Referente ao nível de dor no período inicial da menstruação avaliado através da EVA, foram observados os dados antes da intervenção, e o nível de dor pós menstrual após a realização dos tratamentos. Com o uso da TENS obteve-se os valores de 6,667 ¥2,06 (EVA), na fase inicial da menstruação antes da aplicação da técnica e na fase pós menstrual, após o uso da terapia, houve uma redução para $0,833 \pm 2,04$. Por sua vez, com a manipulação visceral, o nível de dor no período inicial da menstruação foi de 7,000 $\pm 2,38$ e no pós menstrual foi de $0,5729 \pm 1,51$. É possível observar que o uso da manipulação visceral, neste estudo, obteve um resultado melhor, do que no grupo das mulheres que fizeram o uso da TENS, apesar de ambos apresentarem resultados estatisticamente expressivos $(\mathrm{p}<0,05)$, sendo capazes de obter uma melhora significativa da dor. (figura 1). 


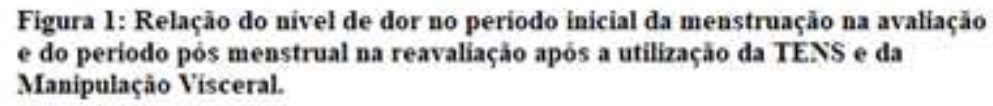

DOR PRE POS TENS

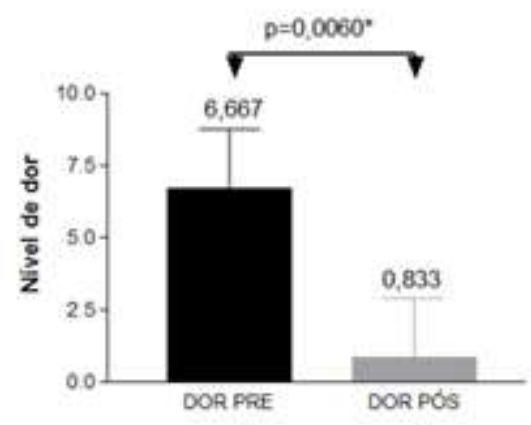

Fonte: Autores (2021)
DOR PRE POS VISCERAL

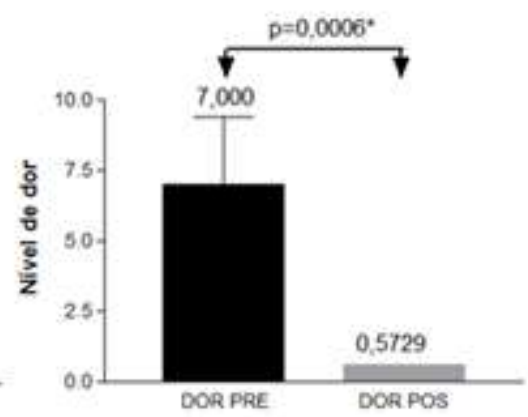

Quanto à média de dor em todo o período menstrual, foram observados os dados na avaliação e na reavaliação das pacientes após a terapia utilizada. Sendo assim, as mulheres que foram tratadas com a TENS na avaliação apresentaram um nível de dor de $9,167 \pm 0,98$ e na reavaliação houve uma redução para 6,333 $\pm 1,86$, como evidenciado na figura 2 . Por sua vez, as mulheres que foram tratadas com a manipulação visceral obtiveram um nível de dor na avaliação de 7,429 $\pm 1,13$ e na reavaliação apresentaram uma diminuição para 4,000 $\pm 2,16$. É possível observar que ambas as técnicas obtiveram um resultado estatisticamente significativo, no entanto a manipulação visceral obteve um valor ainda mais considerável. (p<0,05) (Figura 2).

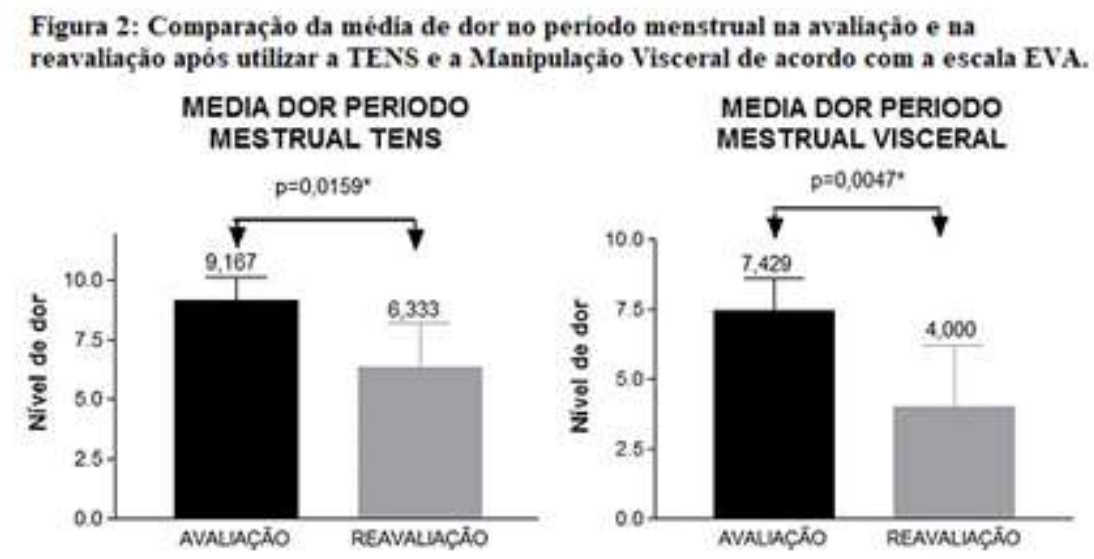

Fonte: Autores (2021)

Ao ser analisado a comparação das médias do nível de dor nas fases do ciclo menstrual antes da aplicação da TENS nas pacientes, foi obtido valores de nível de dor na ovulação de 4,170 \pm 1.94 (EVA); nível de dor antes da menstruação sem ser de fato cólicas menstruais, ou seja, dores de cabeça, dores nas mamas, dores nas pernas de 4,670 $\pm 3,20$; nível de dor na fase inicial da menstruação de 6,670 \2,06; nível de dor até o segundo dia da menstruação e após o mesmo de 9,000 $\pm 1,26$. Enquanto que, após o tratamento houve uma redução para 3,170 $\pm 3,31$ no nível de dor na ovulação, 2,170 $\pm 2,71$ no nível de dor antes da menstruação sem ser cólicas menstruais, 5,840 $\pm 2,99$ no nível de dor na fase inicial da menstruação; 6,840 $\pm 2,13$ no nível de dor até o segundo dia da menstruação e de 3,333 \$3,44 no nível de dor após o segundo dia de menstruação. Apesar de haver uma redução desses valores após o tratamento, não houveram resultados estatisticamente significativos em nenhuma das variáveis apresentadas ( $p>0,05)$, no entanto houve melhora da dor. (Figura 3) 
Figura 3: Comparaçào das médias do nível de dor antes e após a aplicaçảo da TENS durante as fases do ciclo mentrual.

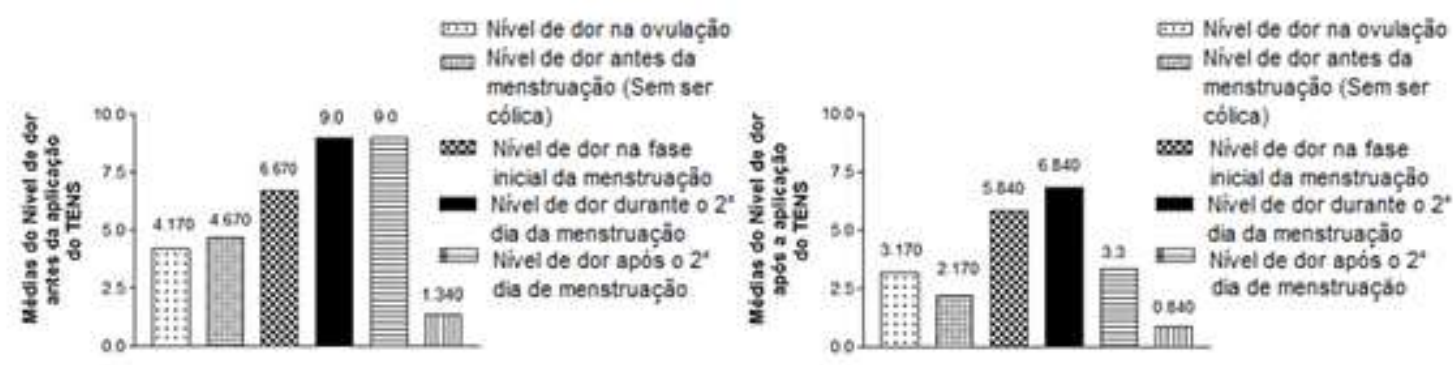

Fonte: Autores (2021)

Por sua vez, ao ser analisada a comparação das médias do nível de dor nas fases do ciclo menstrual antes da Manipulação Visceral, foi possível identificar os valores do nível de dor na ovulação de 4,860 $\pm 2,96$; nível de dor antes da menstruação sem ser cólicas menstruais de 6,430 $\pm 3,82$; nível de dor na fase inicial da menstruação de 7,000 $\pm 2,38$; nível de

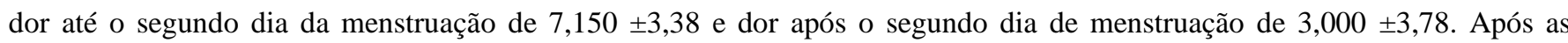
pacientes realizarem o tratamento obteve-se uma diminuição desses valores, sendo eles de 2,150 $\pm 2,47$ no nível de dor na ovulação; 3,430 $\pm 1,81$ no nível de dor antes da menstruação sem ser cólicas menstruais; 3,150 $\pm 1,57$ no nível de dor na fase inicial da menstruação; 4,430 $\pm 2,50$ no nível de dor até o segundo dia da menstruação e de 2,000 $\pm 2,88$ no nível de dor após o segundo dia de menstruação.

Ao compararmos as médias do antes e o depois do tratamento foi possível verificar uma diferença entre eles, no entanto, as variáveis nível de dor antes da menstruação sem ser cólica e nível de dor na fase inicial da menstruação obtiveram resultados estatisticamente expressivos ( $\mathrm{p}<0,005)$, o que valida essa melhora da dor apresentada pelas participantes. (Figura 4).

Figura 4: Comparaçào das médias do nivel de dor antes e após a aplicação da Manipulação Visceral đurante das fases do ciclo menstrual.
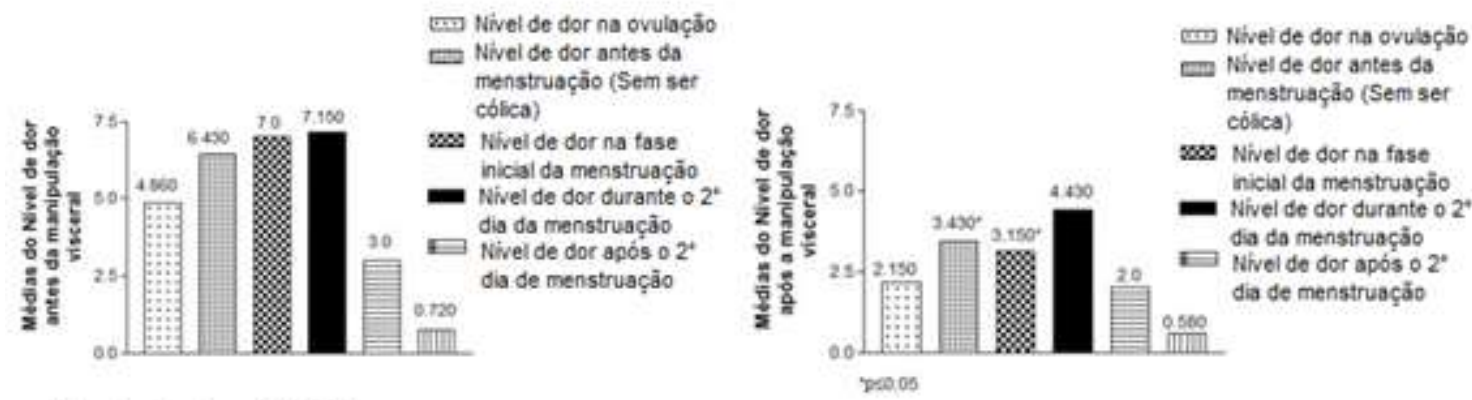

Fonte: Autores (2021)

\section{Discussão}

A dismenorreia primária é uma patologia que afeta a vida de diversas mulheres proporcionando vários desconfortos, sendo o predominante, a dor das cólicas menstruais, que interferem no seu dia a dia antes, durante e após a menstruação. O presente estudo analisou mulheres que possuem esta patologia e observou a melhora da dor causada por ela de acordo com as terapias utilizadas. 
Ao observar a média de idade das mulheres que possuíam cólicas menstruais causadas pela dismenorreia primária, o estudo obteve o valor de $22 \pm 2,00$ anos de idade, dado que corroborou com os estudos de Oliveira et Al. (2012), Rodrigues et Al. (2011) e Canário e Gilly (2019) no qual foram identificados uma média de idade em torno de $20 \pm 2,23$ anos, 19,8 $\pm 3,7$ anos e $23 \pm 2,16$ anos, respectivamente. Sugerindo um possível padrão onde as jovens dessa média de idade têm uma maior tendência a apresentar dismenorreia primária, fator este que pode ser explicado também pela relação do volume uterino com a concentração de prostaglandina, pois, nessa faixa etária ocorre uma alteração desse volume, que na mulher jovem se apresenta como 30-50 $\mathrm{cm}^{3}$, podendo progredir até $90 \mathrm{~cm}^{3}$. Quanto menor o volume, maior a concentração de prostaglandina, causando uma contração mais intensa e gerando mais dor (Soares, 2018).

A fisioterapia possui uma vasta diversidade de recursos que podem ser utilizados no tratamento da dor causada pela dismenorreia primária, como a TENS Burst e a Manipulação Visceral, os quais foram utilizados no presente estudo. De acordo com Monteiro (2009), a TENS Burst quando aplicada com uma frequência de $100 \mathrm{~Hz}$ por um tempo de 30 minutos, em um grupo de mulheres com dismenorreia primária, demonstrou melhora da dor no $3^{\circ}$ mês de tratamento de 45,16\%. Assim como no estudo de Ribeiro et Al. (2020), o tratamento com a TENS Burst em 35 mulheres após 12 atendimentos obteve uma melhora da dor de 7,28 para 3,54 na escala EVA. Dados estes que consolidam os achados do estudo em tela, no qual também houve uma melhora no índice de dor após utilização dos mesmos parâmetros apresentados pelos autores, porém, após o primeiro mês de intervenção, o que sugere uma maior efetividade.

Já no estudo de Karl-Schindler (2006), em uma amostra de 20 pacientes, foi usado um protocolo osteopático com as Técnicas de Mitchell e Manipulação Visceral do útero para o tratamento dos sintomas da dismenorreia primária. Ao término, 50\% das mulheres relataram diminuição da dor na escala EVA de 9 para 2, o que demonstra um efeito positivo da técnica. Assim como, no estudo realizado por Canária e Gilly (2019), em que mulheres com a mesma patologia foram submetidas a um tratamento de manipulação visceral do útero e manipulação visceral do útero associado a terapia craniosacral, obtiveram como resultado, melhora dos sintomas da dismenorreia em ambas as técnicas, apesar da terapia associada apresentar um maior efeito. Ressaltando, que estes estudos evidenciaram a efetividade dessa terapia na população de mulheres com dismenorreia primária, como também foi observado no presente estudo.

Segundo Hedley (2010), quando existe uma alteração da mobilidade característica de uma víscera, gera-se uma aderência nos tecidos próximos ao órgão, causando imobilidade em seus devidos ligamentos. Diante deste fato, é possível afirmar que, havendo uma alteração da mobilidade ou motilidade do útero, o ligamento largo, ligamento útero-ovariano e ligamento redondo do útero sofrerão sobrecargas, podendo levar a uma futura congestão pélvica.

Ao realizar a manipulação da víscera, é esperado que se obtenha uma diminuição das aderências causadas nesses ligamentos, que ajudarão no restabelecimento da produção de prostaglandina, pois haverá uma redução da congestão, proporcionando assim a diminuição das cólicas menstruais (Hedley, 2010). Após os atendimentos realizados no presente estudo, as pacientes obtiveram através da manipulação uma melhora significativa quanto a média da dor durante o seu novo ciclo, em conformidade com os dados apresentados. ${ }^{4,18}$

De acordo com Santos (2020) e Mrugacz (2013), toda mulher apresenta um nível de prostaglandina maior na fase lútea, do que na fase folicular. No entanto, aquelas que possuem dismenorreia primária possuem esse índice ainda mais elevado nesse período. Logo, ao ser realizada a manipulação visceral do útero tende a restabelecer a produção desse composto, proporcionando uma diminuição da dor apresentada nesse período, afirmação esta que corrobora com o achado do presente estudo, em que a fase inicial da menstruação foi uma das que apresentou o maior índice de melhorias utilizando esta técnica. Além disso,observa-se que nesta patologia, a dor vem associada de outros sinais e sintomas, estes que no estudo atual, apresentaram-se também na fase lútea e obtiveram melhora após o tratamento, confirmando a eficiência da técnica. 
Sabendo disso, é possível sugerir que a terapia manual promova uma melhora mais evidente neste estudo, quando comparada com a eletroterapia, apesar de ambas possuírem seus benefícios claros e evidentes. Este resultado pode ser justificado segundo Kramp (2012), pelo fato de, ao realizar a manipulação do útero e do ovário, ocorre uma redução da congestão do sistema linfático na região pélvica, que associado ao viés hormonal, pode ser um dos causadores da dismenorreia primária. Quando se realiza as liberações das restrições fasciais e ligamentares através do tratamento visceral, diminui-se a pressão nos vasos sanguíneos, que causará uma maior eficácia do sistema linfático, que por sua vez, irá auxiliar na restauração do fluxo sanguíneo ideal. Ressaltando que, essa restauração do fluxo, seria indispensável para a normalização da produção de hormônios, havendo assim uma facilitação da sua chegada aos tecidos alvos na cavidade pélvica.

Essa restauração do fluxo sanguíneo fará com que o ácido araquidônico que serve de substrato para via das ciclooxigenases 2 (COX2), que são responsáveis pela síntese de prostaglandinas, que participam de ações metabólicas, como a contração e relaxamento do útero, contarem com sua produção restabelecida (Hilário, 2016). Logo, essa descongestão desempenhada pela manipulação visceral, sugere a normalização da produção hormonal e do próprio ciclo menstrual, fazendo com que haja uma redução da dor mais eficiente, agindo de forma direta no composto causador da patologia (Melo, 2017).

\section{Conclusão}

Após o presente estudo, pode-se concluir que ambas as técnicas apresentaram efeitos benéficos, no entanto a manipulação visceral demonstrou resultados mais positivos, principalmente, na fase inicial da menstruação do ciclo das participantes.

A técnica de manipulação visceral mostrou-se capaz de diminuir a dor dessas mulheres de forma mais eficaz tanto quanto os sintomas apresentados nessa fase específica. No entanto, a redução do número de participantes do estudo e da quantidade de atendimentos realizados, diante a pandemia do Covid-19, limitou os resultados do estudo. Além disso, a literatura escassa sobre o uso dessa técnica, dificultou a construção do mesmo.

Diante do exposto, denota-se a necessidade de serem realizadas mais pesquisas nessa área, para que haja uma base de estudos maior e melhor sobre essa técnica e seus benefícios.

\section{Referências}

Acqua, R. D., \& Bendlin, T. (2015) Dimenorreia. Revista Femina, 43(3):274-276. http://files.bvs.br/upload/S/0100-7254/2015/v43n6/a5327.pdf

Barral, J. P. (2014). Manipulação visceral I. Upledger Brasil.

Canário, M. B., \& Gilly, D. (2019) Efeitos da terapia craniossacral e a mobilização uterina no alívio da dismenorreia primária: um estudo piloto. Revista FisiSenectus, 7(2):12-22. https://bell.unochapeco.edu.br/revistas/index.php/fisisenectus/article/view/5050

Claydon, L. S. Kannan, P. (2014) Some physioterapy treatments may relieve menstrual pain in women with primary dysmenorrhea: a systematic review. Journal of Physiotherapy, Australia, 60(1):13-21.: https://pubmed.ncbi.nlm.nih.gov/24856936/

Ciena, A. P., Gatto, R., Pacini, V. C., Picanço, V. V., Magno, I. M. N., \& Loth, E. A. (2008) Influência da intensidade da dor sobre as respostas nas escalas unidimensionais de mensuração da dor em uma população de idosos e de adultos jovens. Semina: Ciências Biológicas e da Saúde, Londrina, 29(2):201-212. http://www.uel.br/revistas/uel/index.php/seminabio/article/view/3467

Hedley, G. (2010) Notes on visceral adhesions as fascial pathology. Journal of bodywork and movement therapies, 14(1):255-261. https://www.sciencedirect.com/science/article/pii/S1360859209001296

Hilário, M. O. T., Terreri, M. T., \& Len, C. A. (2016) Nonsteroidal anti-inflammatory drugs: cyclooxygenase 2 inhibitors. Jornal de Pediatria, 82(5):206-212. https://www.scielo.br/j/jped/a/xw7Py6gTnnHtvC3z3sJ4XTm/?lang=en\&format=html

Iacovides, S., Avidon, I., \& Baker F. C. (2015) What we know about primary dysmenorrhea today: a critical review. Human Reproduction Update, 21(6):762777. https://academic.oup.com/humupd/article-abstract/21/6/762/628858

Karl-Schindler, H. (2006) Osteopathic treatment of primary dismenorrhoea: possible consequences. Master thesis (Osteopathy) at the Donau Universität Krems, Obendorf, Austria. https://www.osteopathic-research.com/files/original/e59c06c92ede50d1d1307a6491e6f63cf0534a76.pdf 
Kramp, M. E. (2012) Combined Manual Therapy Techni-ques for the Treatment of Women With Infertility: A Case Series, The jornal of the american osteopathic association, 112(10):680-684. https://www.degruyter.com/document/doi/10.7556/jaoa.2012.112.10.680/html

Lopes, K. N. (2016) Avaliação dos limiares sensitivo e doloroso em mulheres com dismenorreia primaria moderada ou grave. Dissertação de Mestrado, Faculdade de medicina de Ribeirão Preto, Universidade de São Paulo, Ribeirão Preto. 10.11606/D.17.2016.tde-26082016-112528.

Melo, B. A. P., Zago, C. C., \& Biffe, B. G. (2017) Atuação da fisioterapia pélvica em pacientes com dismenorreia primária. Trabalho de conclusão de curso, Centro Universitário Católico Salesiano Auxilium de Araçatuba. http://docplayer.com.br/79659492-Atuacao-da-fisioterapia-pelvica-em-pacientes-comdismenorreia-primaria.html

Monteiro, A. M. (2009). Estudo comparativo da Tens Burst e da massagem do tecido conjuntivo no tratamento de dismenorreia primária. Trabalho de conclusão de curso, Faculdade Avila, Universidade do Estado do Pará., Santarém. https://portalbiocursos.com.br/ohs/data/docs/32/108__Estudo_comparativo_da_Tens_Burst_e_da_Massagem_do_tecido_conjuntivo_no_tratamento_de_DismenorrYia_primYria.pdf

Mrugacz, G., Grygoruk, C., Sueczynski, P., Grusza, M., Bolkun, I., \& Pietrewicz, P. (2013). Etiopatogeneza zespołu bolesnego miesiączkowania. Developmental Period Medicine. 17(1):85-89. https://www.osteopathic-research.com/files/original/e59c06c92ede50d1d1307a6491e6f63cf0534a76.pdf

Paulino L. S. S. Teles, A. \& Lordêlo, P. (2014) Estimulação elétrica nervosa transcutânea na dismenorreia primária. Revista Pesquisa em Fisioterapia, 4(1):47-54 https://www5.bahiana.edu.br/index.php/fisioterapia/article/view/325

Reis, A. M. Souza, E. S. Bueno, M. A. F. \& Costa, A. C. S. (2016) A importância da fisioterapia no tratamento da dismenorreia primaria: um estudo comparativo. Revista Ciências do Unisalesiano Lins-SP. 7(15):502-514. http://www.salesianolins.br/universitaria/artigos/no15/artigo35.pdf

Ribeiro, G. O., Souza, F. S., Trindade, G. M. C., Barbosa, G. S. V., Alves, M. H. F., \& Gomes, V. M. S. A. (2020) Eletroanalgesia na redução do quadro álgico em universitárias portadoras de dismenorreia. Brazilian Journal of Development.6(7): 47918-47932. https://www.brazilianjournals.com/index.php/BRJD/article/view/12414

Rodrigues, A. C., Gala, S., Neves, A., Pinto, C., Meirelles, C., Frutuoso, C., \& Vitor, M. E. (2011) Dismenorreia em adolescentes e jovens: Prevalência, factores associados e limitações na vida diária. Acta Médica Portuguesa. 24(2): 383-392. https://www.actamedicaportuguesa.com/revista/index.php/amp/articl e/viewFile/1477/1063

Santos, L. B. (2020) Dismenorreia e funcionalidade em mulheres adultas do nordeste brasileiro. Dissertação de mestrado, Faculdade de Ciências da Saúde do Trairi, Universidade Federal do Rio Grande do Norte. Santa Cruz. https://repositorio.ufrn.br/handle/123456789/30063

Soares, N. B. (2018). Recursos fisioterapêuticos utilizados para o tratamento da dismenorreia primária: revisão de literatura. Dissertação de Pós Graduação, Centro Universitário de Brasília. https://repositorio.uniceub.br/jspui/bitstream/235/12273/1/51500733.pdf

Starkey, C. (2001). Recursos terapêuticos em fisioterapia. Manole. 